\title{
Photometric Variability of Four Coronally Active Stars
}

\author{
J. C. Pandey ${ }^{1}$, K. P. Singh ${ }^{2}$, R. Sagar ${ }^{1,3} \&$ S. A. Drake ${ }^{4}$ \\ ${ }^{1}$ State Observatory, Naini Tal 263 129, India. \\ ${ }^{2}$ Tata Institute of Fundamental Research, Mumbai, India. \\ ${ }^{3}$ Indian Institute of Astrophysics, Bangalore, India. \\ ${ }^{4}$ Code 668, LHEA, NASA/Goddard Space Flight Center, Greenbelt, MD 20771, USA.
}

\begin{abstract}
We present photometric observations of four stars that are optical counterparts of soft X-ray/EUV sources, namely 1ES 0829+15.9, 1ES0920-13.6, 2RE J110159+223509 and 1ES 1737+61.2. We have discovered periodic variability in two of the stars, viz., MCC 527 (1ES 0829+15.9; Period $=0^{d} .828 \pm 0.0047$ ) and HD 81032 (1ES 0920-13.6; Period $=\sim 57.02 \pm 0.560$ days). HD $95559(2 \mathrm{RE} \mathrm{J110159+223509)}$ is found to show a period of $3^{d}$. HD 160934 (1ES1737+61.2) also shows photometric variability but needs to be monitored further for finding its period. These stars most likely belong to the class of chromospherically active stars.
\end{abstract}

Key words. Stars—variable, optical photometry, X-rays-source.

\section{Introduction}

A number of soft X-ray sources have recently been discovered in X-ray surveys with the Einstein and the ROSAT observatories and found to be associated with bright latetype stars. Many of these stars have not been studied in detail for their chromospheric and coronal activity, and their nature is not fully understood. As a result these are also not properly classified. We have carried out photometric observations of four such stars. Three of these: MCC 527, HD 81032, and HD 160934, were identified as likely optical counterparts of X-ray sources 1ES 0829+15.9, 1ES 0920-13.6, 1ES 1737+61.2 seen in the Einstien's IPC slew survey (Elvis et al. 1992). Another star, HD 95559, has been detected as an extreme ultraviolet (EUV) source named 2RE J110159+223509 by Mason et al. (1995). Optical spectra of these stars show chromospheric activities based on $H_{\alpha}$ and/or CaII, $\mathrm{H}$ and $\mathrm{K}$ emission lines. Table 1 lists the general information about these stars. Their X-ray, optical and radio properties, suggest that these may belong to various classes of coronally active stars.

\section{Observations and data reductions}

The BVR photometric observations were carried out using a CCD camera at f/13 Cassegrain focus of the 104-cm Sampurnanand Telescope at the State Observatory, Naini Tal. The CCD system consists of $24 \times 24 \mu^{2}$ size pixel, having $2048 \times 2048$ pixels. To improve the signal-to-noise ratio the observations have been taken in a binning 
Table 1. General information on the program stars. $\Delta m$ is difference in magnitude between the maximum and minimum of light curve in a filter and $\sigma$ is the standard deviation of the difference in magnitude of comparison stars.

\begin{tabular}{cccccccc}
\hline X-ray source & $\begin{array}{c}\text { Optical } \\
\text { counterpart }\end{array}$ & $\begin{array}{c}V \\
(\mathrm{mag})\end{array}$ & $\begin{array}{c}\text { Co-ordinate } \\
(2000)\end{array}$ & Filter & $\begin{array}{c}\Delta m \\
(\mathrm{mag})\end{array}$ & $\begin{array}{c}\sigma \\
(\mathrm{mag})\end{array}$ & $\Delta m / \sigma$ \\
\hline 1ES 0829+15.9 & MCC 527 & \multirow{2}{*}{10.41} & $\alpha=08^{h} 32^{m} 30^{s}$ & $\mathrm{~B}$ & 0.111 & 0.007 & 14.1 \\
& & & $\delta=15^{0} 49^{\prime} 26^{\prime \prime}$ & $\mathrm{V}$ & 0.114 & 0.006 & 17.9 \\
& & & & $\mathrm{R}$ & 0.103 & 0.007 & 14.7 \\
\hline 1ES 0920-13.6 & \multirow{2}{*}{$\mathrm{HD} \mathrm{81032}$} & \multirow{2}{*}{9.02} & $\alpha=09^{h} 22^{m} 53^{s}$ & $\mathrm{~B}$ & 0.300 & 0.025 & 12 \\
& & & $\delta=-13^{0} 49^{\prime} 43^{\prime \prime}$ & $\mathrm{V}$ & 0.284 & 0.014 & 19.7 \\
& & & & $\mathrm{R}$ & 0.261 & 0.014 & 18.4 \\
\hline WFC & \multirow{2}{*}{ HD 95559 } & \multirow{2}{*}{8.96} & $\alpha=11^{h} 02^{m} 02^{s}$ & $\mathrm{~B}$ & 0.080 & 0.012 & 6.7 \\
J1102.0+2235 & & & $\delta=22^{0} 35^{\prime} 45^{\prime \prime}$ & $\mathrm{V}$ & 0.076 & 0.012 & 6.3 \\
& & & & $\mathrm{R}$ & 0.060 & 0.009 & 6.7 \\
\hline 1ES 1737+61.2 & HD 160934 & \multirow{2}{*}{10.29} & $\alpha=17^{h} 38^{m} 30^{s}$ & $\mathrm{~B}$ & 0.116 & 0.012 & 9.7 \\
& & & $\delta=61^{0} 15^{\prime} 09^{\prime \prime}$ & $\mathrm{V}$ & 0.111 & 0.014 & 8.0 \\
& & & & $\mathrm{R}$ & 0.080 & 0.009 & 8.9 \\
\hline
\end{tabular}

mode of $2 \times 2$ pixel $^{2}$, where each super pixel corresponds to $0.72 \times 0.72 \operatorname{arcsec}^{2}$. The CCD covers a field of view $\sim 13 \times 13$ arcminute $^{2}$.

Multiple CCD frames were taken with the exposure time ranging from 2 to 120 secs depending on the seeing conditions and the filters used. A number of bias and twilight flat field frames were also taken during the observing run. The frames were cleaned employing the IRAF/MIDAS software. The differential magnitude of the stars were determined by aperture photometry.

\section{Analysis and light curve}

Three comparison stars for each program star (2 for HD 95559), having magnitude and colour similar to the program star and in the same CCD frame, were used to check for the variability in the program stars. Pair wise differential magnitudes of the comparison stars were computed and the standard deviations $(\sigma)$ examined. In each case, the standard deviations were found to be nearly equal. Further analysis was carried out with only one of the comparison stars, closest to the program star. The ratio $\Delta m / \sigma$ indicates that all the program stars show statistically significant variability. In each filter, $\Delta m$, the difference between the maxima and the minima of the light curves was also determined. The light curves were analysed for periodicity using the LombScragle periodigram method in Starlink's Period software, and by fitting a sinusoidal function using the least square deviation method. Results from this analysis for each variable star are given below.

\section{$3.11 E S 0829+15.9($ MCC 527)}

Light curves obtained for MCC 527 in the B,V and R filters are shown in Fig. 1 (left panel). MCC 527 is a K8 spectral type star (A. V. Kazarovets et al. 1999), and is also listed as an unsolved variable (FR Cnc) in the Hipparcos catalogue (Perryman et al. 1997). Measurements with the Hipparcos satellite (Perryman et al. 1997) provide 

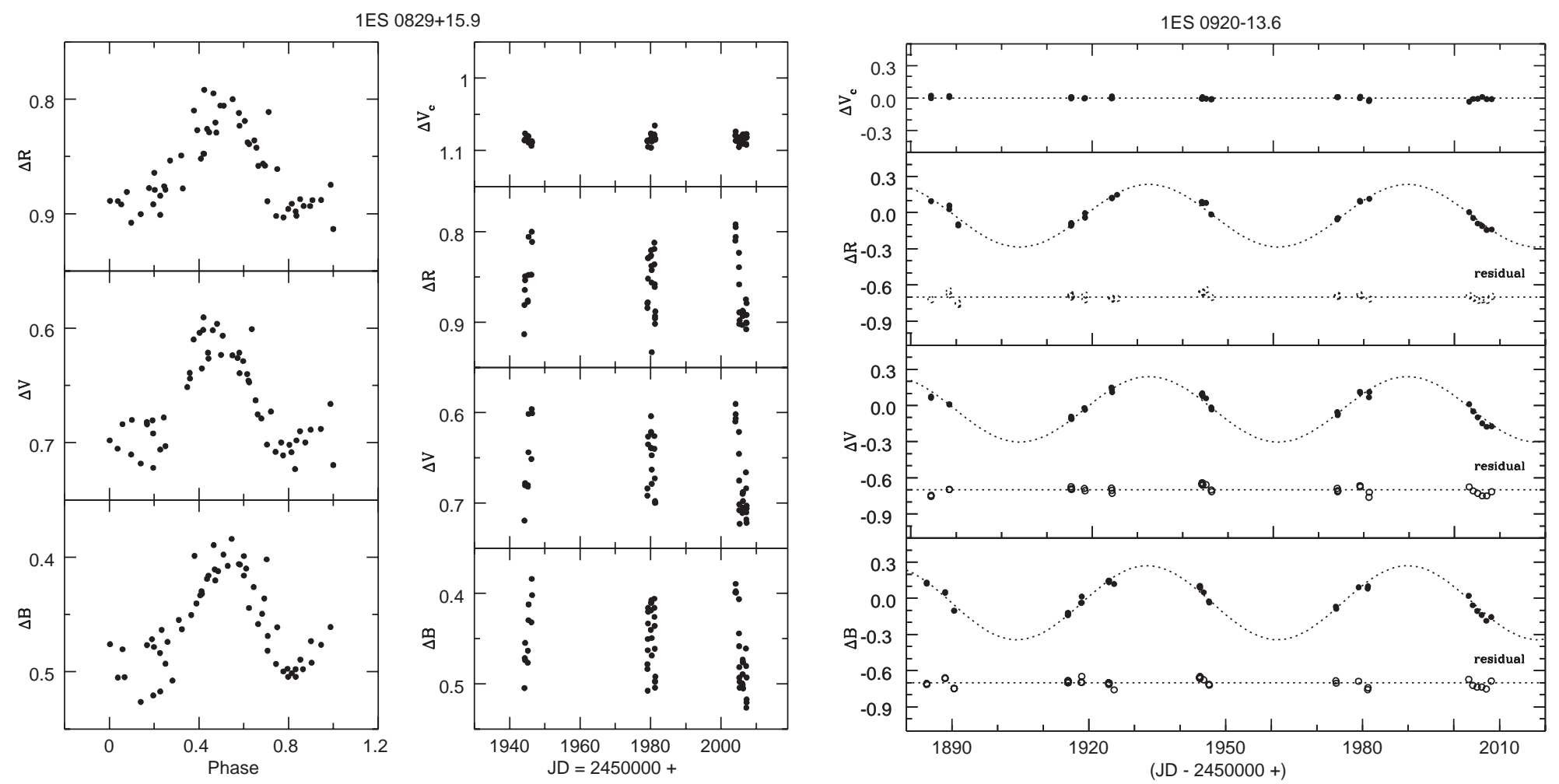

Figure 1. Left panel: Differential $B, V, R$ magnitudes of MCC 527(1ES 0829+15.9) from February - April 2001 observing season against phase, and against Julian date. Right panel: Differential $B, V$ and $R$ light curve of the star HD 81032 (1ES 0920-13.6), observed during $2000-2001$. 
the basic parameters of this star: $V=10.41 \mathrm{mag},(B-V)=1.16 \pm 0.997 \mathrm{mag}$, $(V-I)=2.64 \pm 0.49 \mathrm{mag}, V_{\max }=10.276 \mathrm{mag}, V_{\min }=10.47 \mathrm{mag}$, variability magnitude $(\Delta V)=0.172 \pm 0.043 \mathrm{mag}$ and a parallax of $30.24 \pm 2.03$ milliarcsec which implies a distance of $33_{-2}^{+2} \mathrm{pc}$ and a visual absolute magnitude of $\sim 7.8 \mathrm{mag}$. We have discovered a period of $\sim 0.8281 \pm 0.0027$ days in the star. Fig. 1 (left panel) shows the light curves folded with this period.

\subsection{IES 0920-13.6 (HD 81032)}

$B, V$ and $R$ light curves of HD 81032, optical counterpart of 1ES 0920-13.6, are shown in the right panel of Fig. 1. The basic parameters of HD 81032 are: $V=$ $9.019 \pm 0.023 \mathrm{mag},(B-V)=1.033 \pm 0.023 \mathrm{mag}$ and a parallax of 6.6 milliarcsec (Hog et al. 1997). Our period search finds that the best periods in the $B, V$ and $R$ light curves $57.100 \pm 0.280,57.035 \pm 0.371$ and $57.061 \pm 0.313$ days respectively and the corresponding amplitude are $0.300 \pm 0.034,0.272 \pm 0.037$ and $0.260 \pm 0.031$ respectively. The mean period thus found is $57.065 \pm 0.560$ days. If confirmed, this will be a new periodic variable. The best fit sinusoidal curves, using the least square deviation, are shown in the right panel of Fig. 1.

\section{$3.3 H D 95559$}

$B, V$ and $R$ light curves of HD 95559 observed by us are shown in the left panel of Fig. 2. HD $95559=$ BD $23^{0} 2297$ has recently been shown to be a double-lined spectroscopic binary with an orbital period of 1.5260 , and with a photometric period of 1.5264 days indicating that the rotation in HD 95559 is tightly synchronised to the orbital motion (Fekel \& Henry 2000). Previous reports that this system has a 2.9 day photometric period (Jeffries et al. 1994; Strassmeier et al. 2000) appear to have been detections of the 1-day alias of the 1.526 day orbital period. According to the Hipparacos catalogue (Perryman et al. 1997) the basic parameters of this star are: $V=8.96 \mathrm{mag},(B-V)=0.87 \pm 0.026 \mathrm{mag},(V-I)=0.88 \pm 0.02 \mathrm{mag}, V_{\max }=9.040$ mag, $V_{\min }=9.158$, mag variability magnitude $(\Delta V)=0.095 \pm 0.025$ and parallax $=18.43 \pm 1.19$ milliarcsec which gives a distance of $54_{-3}^{+4} \mathrm{pc}$ and absolute magnitude of $\sim$ 5.3. Its spectral class is K1V (Fekel \& Henry 2000). A combination of the sinusoidals $\left(f(x)=a_{0}+a_{1} \cos \left(2 \pi f\left(x+l_{1}\right)\right)+a_{2} \cos \left(2 \pi f\left(x+l_{2}\right)\right)+a_{3} \sin (2 \pi f(x+\right.$ $\left.\left.\left.l_{3}\right)\right)+a_{4} \sin \left(2 \pi f\left(x+l_{4}\right)\right)\right)$ was fitted using the least square deviation method in each of the $B, V$ and $R$ light curves (left panel of Fig. 2) which show primary and secondary minima. The period was determined by fitting a parabola to the two consecutive primary minimas and is found to be $3^{d} .03 \pm 0.02$, consistent with the 1-day alias of the orbital period.

\section{$3.41 E S 1737+61.2(H D 160934)$}

Light curves obtained for HD 160934 in the $B, V$ and $R$ filters are shown in Fig. 2 (right panel). HD 160934 is a flare star (Gershberg et al. 1999) of spectral type K8V. Its basic parameters are as follows: $V=10.29 \mathrm{mag},(B-V)=1.591 \pm 0.4 \mathrm{mag}$, $(V-I)=2.58 \pm 0.91 \mathrm{mag}, V_{\max }=10.21 \mathrm{mag}, V_{\min }=10.36 \mathrm{mag}$ (Hipparcos catalogue). The parallax of $40.75 \pm 12.06$ milliarcsec provides a distance of $25_{-6}^{+10}$ pc and absolute visual magnitude of 8.4. The observed points shown in Fig. 2 are not 

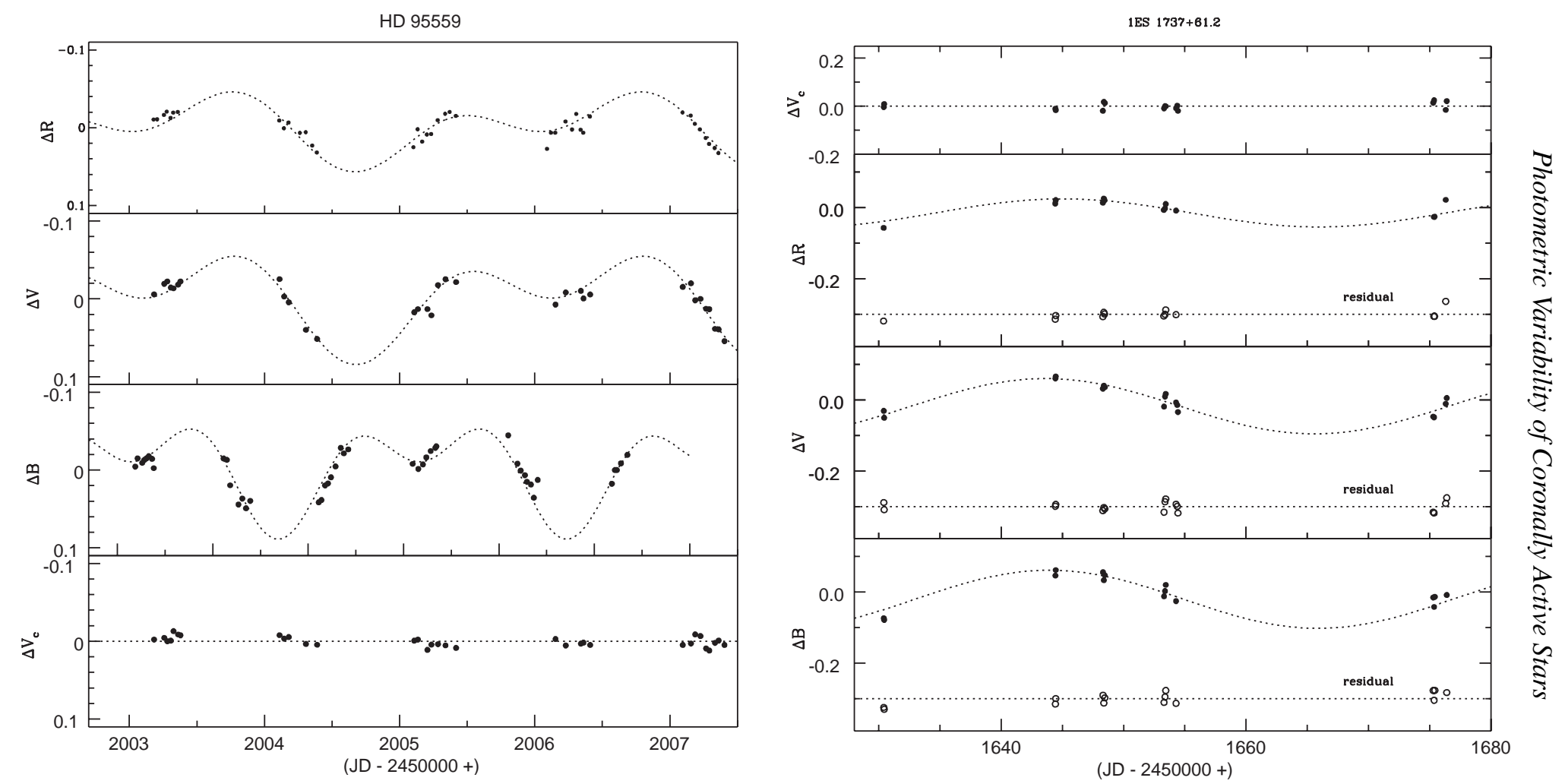

Figure 2. Left panel: $B, V$ and $R$ light curves of HD 95559 as observed in April 3-8, 2001. Right panel: $B, V$ and $R$ light curves of HD 160934 (1ES $1737+61.2)$ as observed during the year 2000 . 
sufficient for finding a reliable period, however, a sinusoidal curve is fitted using the method of the least square deviations for each of the $B, V$ and $R$ light curves. The best period found in $B, V$ and $R$ light curves is $43.918 \pm 0.0154,43.931 \pm 0.012$ and $41.719 \pm 0.0035$ respectively, giving a mean value of $43.182 \pm .040$ days. The inferred period seems rather long for an active late K dwarf considering that $v \sin i=16.4 \mathrm{~km} / \mathrm{s}$ for this star (Fekel 1997), and assuming that $\mathrm{R}=0.6 \mathrm{R}_{/ \mathrm{odot}}$, the maximum period (for $\sin i=1.0$ ) is 1.85 days. More extensive observations are required for this star.

\section{Summary}

Optical photometry of the counterparts of four soft X-ray sources has led to the discovery of periods in two of them, viz., MCC $527\left(\mathrm{P}=0^{d} .828 \pm 0.0047\right)$ and HD 81032 $(\mathrm{P}=\sim 57.065 \pm 0.560$ days $)$. The observed variability appears to be of a different type in each source. We have also found the stars HD 95559 and HD 160934 to be variable. We plan to monitor these objects for longer time periods in three different filters, and to carry out spectroscopic observations, to understand the nature of variability in these stars. Stars MCC 527 and HD 81032 could belong to the short-period and long-period RSCVn class of objects, respectively.

\section{References}

Elvis, M. et al. 1992, ApJS, 80, 257.

Fekel, F. C. 1997, PASP, 109, 514.

Fekel, F. C., Henry, G. W. 2000, AJ, 120, 3265.

Gershberg, R. E. et al. 1999, A\&AS, 193, 555.

Hog, E. et al. 1997, $A \&$ \& A, 323, L57.

Jeffries, R. D. et al. 1994, IBVS Number 4091.

Kazarovets, A. V. et al. 1999, IBVS No. 4659.

Mason, K. O. et al. 1995, MNRAS, 274, 1194.

Perryman, M. A. et al. 1997, A \& A, 323, L49.

Strassmeier, K. G. et al. 2000, A \& AS, 142, 247. 